\title{
Editorial
}

\section{Pressure-Induced Phase Transformations}

\author{
Daniel Errandonea \\ Departamento de Física Aplicada-ICMUV, Universitat de València, Calle Dr. Moliner 50, 46100 Burjassot, Spain; \\ daniel.errandonea@uv.es \\ Received: 7 July 2020; Accepted: 9 July 2020; Published: 10 July 2020 \\ check for \\ updates
}

The study of phase transitions in solids under high pressure conditions is a very active and vigorous research field. In recent decades, thanks to the development of experimental techniques and computer simulations, a plethora of important discoveries has been made under high-pressure conditions. Many of the achievements accomplished in recent years affect various research fields, from solid-state physics to chemistry, materials science, and geophysics. They not only contribute to a deeper understanding of solid-sold phase transitions but also to a better understanding of melting under compression.

This Issue collects thirteen contributions, starting with the paper of Bing Li et al. [1]. This paper presents a Raman and X-ray diffraction study of $C_{60}$ fullerene. The authors showed that $C_{60}$ underwent a phase transition from a face-centered cubic structure to a single cubic structure at around $0.3 \mathrm{GPa}$. They also report evidence of dimerization of $\mathrm{C}_{60}$ at about 3.2 GPa.

In the second article, Andreas Tröster et al. [2] introduce a new version of the Landau theory, which is based on symmetry-adapted finite strains, which results in a substantial simplification of the original formulation. These authors apply their theoretical development to the high-pressure phase transition of the perovskite $\mathrm{KMnF}_{3}$, characterizing in detail the cubic-tetragonal transition that occurs around 3.4 GPa.

In recent years, hydrated sulfates and their high-pressure behavior have attracted a large amount of interest due to their great importance in exploring the interior structure of icy satellites, such as Europa, Ganymede, and Callisto. In their work, Linfei Yang et al. [3] studied epsomite $\left(\mathrm{MgSO}_{4} \cdot 7 \mathrm{H}_{2} \mathrm{O}\right)$, a representative of magnesium-bearing hydrated sulfates. The reported results could contribute to understanding the interior structure, composition, and physical properties of the icy satellites. The most interesting finding of this work is the observation that epsomite undergoes a three-step dehydration reaction under high pressure, and its dehydration temperature gradually increases with pressure.

Melting under compression is one of the most challenging subjects of high-pressure studies. In this Issue, Samuel Baty et al. [4] report density-functional studies of molybdenum (Mo) and tungsten (W), two metals with a high melting temperature. In particular, they demonstrate the topological equivalence of both Group 6B elements. Phase diagrams have been extended to 2000 (Mo) GPa and 2500 (W) GPa, respectively. For both metals, the authors propose the existence of two solid structures: the ambient body-centered cubic and a high-pressure double hexagonal close-packed. The solid-solid transition occurs at $660 \mathrm{GPa}$ in Mo and $1060 \mathrm{GPa}$ in W.

In the subsequent contribution, Raquel Chuliá-Jordán et al. [5] reported laser-heating diamond-anvil cell studies of $\mathrm{CO}_{2}$ and carbonate. They present experimental data that evidences the chemical reactivity between rhenium $(\mathrm{Re})$ and tungsten $(\mathrm{W})$ with carbon dioxide $\left(\mathrm{CO}_{2}\right)$ and carbonates at temperatures above $1300{ }^{\circ} \mathrm{C}$ and pressures above $6 \mathrm{GPa}$. Metal oxides and diamond are identified as reaction products. Recommendations to minimize non-desired chemical reactions in high-pressure high-temperature experiments are given.

Another high-pressure and high-temperature study is the contribution by Ezenwa and Secco [6]. The study is focused on the electrical resistivity and thermal conductivity behavior of iron ( $\mathrm{Fe}$ ) at core conditions. The results of such studies are important for understanding planetary interior thermal 
evolution as well as characterizing the generation and sustainability of planetary dynamos. The study also discusses the behavior of $\mathrm{Fe}$, cobalt (Co), and nickel (Ni), at the solid-liquid melting transition. In particular, the authors report the thermal conductivity difference on the solid and liquid sides of Mercury's inner core boundary and discuss the implications of their findings on the modeling of the adiabatic heat flow of on the inner core side.

An interesting problem is the behavior of organic compounds under high-pressure. In such compounds, a small pressure of the order of a few GPa could have drastic consequences. In the contribution to this Issue, Mahesta and Mochizuki [7] investigate, using molecular dynamics simulations, the spontaneous homogeneous melting of benzene $\left(\mathrm{C}_{6} \mathrm{H}_{6}\right)$ under a pressure of 1.0 GPa. They propose the existence of an apparent stepwise transition via a metastable crystal phase, unlike the direct melting observed at ambient pressure. The transition to the metastable phase is achieved by rotational motions, without the diffusion of the center of mass of benzene. These results could open the door to interesting novel findings on the behavior hydrocarbons under compression.

In another original article, Enrico Bandiello et al. [8] studied the high-pressure behavior of zircon-type $\mathrm{NdVO}_{4}$. In particular, these authors report on optical spectroscopic measurements in pure $\mathrm{NdVO}_{4}$ crystals at pressures up to $12 \mathrm{GPa}$. They correlated the influence of pressure on the crystal structure and pressure-induced structural transition with the high-pressure behavior of the fundamental absorption band gap and the $\mathrm{Nd}^{3+}$ absorption bands. The experiments indicate that a phase transition takes place near $5 \mathrm{GPa}$. Bandiello et al. also have also determined the pressure dependence of the band-gap and discussed the behavior of the $\mathrm{Nd}^{3+}$ absorption lines under compression. Important changes in the optical properties of $\mathrm{NdVO}_{4}$ occur at the phase transition, which, according to Raman measurements, corresponds to a zircon to monazite phase change. In particular, in these conditions a collapse of the band gap occurs, changing the color of the crystal. The results of the study are analyzed in comparison with those deriving from previous studies on $\mathrm{NdVO}_{4}$ and related vanadates.

In addition to the eight original research articles described above, this Special Issue also includes five review articles. In the first one, Anzellini and Boccato [9] present an extensive review of the laser-heated diamond-anvil cell technique for university laboratories and synchrotron applications. In the last two decades, the laser-heated diamond anvil cell method, combined with different characterization techniques, has become an extensively used tool for studying pressure-temperature-induced evolution of various physical and chemical properties of materials. In their review, the authors present and discuss the general challenges associated with the use of laser-heated diamond-anvil cells. This discussion is combined with examples of the recent progress in the use of this tool, combined with synchrotron X-ray diffraction and absorption spectroscopy.

Another article focused on recent developments of experimental techniques is the one of Popov et al. [10]. This review is devoted to synchrotron X-ray radiation Laue diffraction, a widely used diagnostic technique for characterizing the microstructure of materials. The authors describe in detail the current status of this powerful technique, including experimental routines and data analysis. They also present results from some case studies and a description of the new experimental setup at the High-Pressure Collaborative Access Team (HPCAT) facility at the Advanced Photon Source, specifically dedicated for in situ and in operando microstructural studies by Laue diffraction under high pressure.

In another of the reviews, Denis Rychov [11] presents a description of the progress made by him and his collaborators on computational studies of high-pressure phase transitions in molecular crystals. The advantages and disadvantages of different approaches are discussed, and the interconnection of experimental and computational methods is highlighted. Finally, challenges and possible ways for progress in high-pressure phase transition research of organic compounds are briefly discussed.

Biesner and Uykur authored another review paper [12], which focused on quantum spin liquids, which are prime examples of strongly entangled phases of matter with unconventional exotic excitations. In particular, they discussed how strong quantum fluctuations prohibit the freezing of the spin system. They also discussed frustrated magnets, which are candidates to search for the quantum spin liquids. 
The main topic of the review is the ability of pressure to influence the magnetic phases. The authors review experimental progress in the field of pressure-tuned magnetic interactions, showing that chemical or external pressure is a suitable parameter to create exotic states of matter.

In their recent article, Prof. Manjon et al. [13] conduct an extensive review of the progress they have made in recent years on pressure-induced phase transition in sexquioxides. These compounds constitute a large subfamily of $\mathrm{ABO}_{3}$-type compounds, which have many different crystal structures due to their large diversity of chemical compositions. They are very important for Earth and Materials Sciences, thanks to their presence in our planet's crust and mantle, and their wide variety of technological applications. Recent discoveries, hot spots, controversial questions, and future directions of research are highlighted in the article.

In summary, the articles presented in this Special Issue are representative of some of the lines of a topic as broad as high-pressure research as well as of its importance in different scientific fields, and cover aspects including structural, electronic, and magnetic transitions. They touch on the latest advancements in several aspects related to the behavior of matter under high-pressure. The included papers show that impressive progress has been made recently on high-pressure research.

Conflicts of Interest: The authors declare no conflict of interest.

\section{References}

1. Li, B.; Zhang, J.; Yan, Z.; Feng, M.; Yu, Z.; Wang, L. Pressure-Induced Dimerization of $\mathrm{C}_{60}$ at Room Temperature as Revealed by an In Situ Spectroscopy Study Using an Infrared Laser. Crystals 2020, 10, 182. [CrossRef]

2. Tröster, A.; Schranz, W.; Ehsan, S.; Belbase, K.; Blaha, P. Symmetry-Adapted Finite Strain Landau Theory Applied to $\mathrm{KMnF}_{3}$. Crystals 2020, 10, 124. [CrossRef]

3. Yang, L.; Dai, L.; Li, H.; Hu, H.; Hong, M.; Zhang, X. The Phase Transition and Dehydration in Epsomite under High Temperature and High Pressure. Crystals 2020, 10, 75. [CrossRef]

4. Baty, S.; Burakovsky, L.; Preston, D. Topological Equivalence of the Phase Diagrams of Molybdenum and Tungsten. Crystals 2020, 10, 20. [CrossRef]

5. Chuliá-Jordán, R.; Santamaría-Pérez, D.; Marqueño, T.; Ruiz-Fuertes, J.; Daisenberger, D. Oxidation of High Yield Strength Metals Tungsten and Rhenium in High-Pressure High-Temperature Experiments of Carbon Dioxide and Carbonates. Crystals 2019, 9, 676. [CrossRef]

6. Ezenwa, I.C.; Secco, R.A. Fe Melting Transition: Electrical Resistivity, Thermal Conductivity, and Heat Flow at the Inner Core Boundaries of Mercury and Ganymede. Crystals 2019, 9, 359. [CrossRef]

7. Mahesta, R.; Mochizuki, K. Stepwise Homogeneous Melting of Benzene Phase I at High Pressure. Crystals 2019, 9, 279. [CrossRef]

8. Bandiello, E.; Sánchez-Martín, J.; Errandonea, D.; Bettinelli, M. Pressure Effects on the Optical Properties of $\mathrm{NdVO}_{4}$. Crystals 2019, 9, 237. [CrossRef]

9. Anzellini, S.; Boccato, S. A Practical Review of the Laser-Heated Diamond Anvil Cell for University Laboratories and Synchrotron Applications. Crystals 2020, 10, 459. [CrossRef]

10. Popov, D.; Velisavljevic, N.; Somayazulu, M. Mechanisms of Pressure-Induced Phase Transitions by Real-Time Laue Diffraction. Crystals 2019, 9, 672. [CrossRef]

11. Rychkov, D.A. A Short Review of Current Computational Concepts for High-Pressure Phase Transition Studies in Molecular Crystals. Crystals 2020, 10, 81. [CrossRef]

12. Biesner, T.; Uykur, E. Pressure-Tuned Interactions in Frustrated Magnets: Pathway to Quantum Spin Liquids? Crystals 2020, 10, 4. [CrossRef]

13. Manjón, F.J.; Sans, J.A.; Ibáñez, J.; Pereira, A.L.J. Pressure-Induced Phase Transitions in Sesquioxides. Crystals 2019, 9, 630. [CrossRef] 